\title{
Hyperbaric Oxygen Therapy of Ischemic Cranial Skin Flap: Case Report and Review of the Literature
}

\author{
Fassil B. Mesfin ${ }^{1}$, Matthew R. Burton², Ruben A. Ngnitewe Massa ${ }^{3}$, Jeffery S. Litt ${ }^{2}$ \\ ${ }^{1}$ Divsion of Neurological Surgery, One Hospital Drive, Columbia, USA \\ ${ }^{2}$ Divsion of Acute Care Surgery, Department of Surgery at One Hospital Drive, Columbia, USA \\ ${ }^{3} \mathrm{MU}$ School of Medicine, One Hospital Drive, Columbia, USA \\ Email: *mesfinf@health.missouri.edu
}

How to cite this paper: Mesfin, F.B., Burton, M.R., Massa, R.A.N. and Litt, J.S. (2017) Hyperbaric Oxygen Therapy of Ischemic Cranial Skin Flap: Case Report and Review of the Literature. Case Reports in Clinical Medicine, 6, 250-254.

https://doi.org/10.4236/crcm.2017.610028

Received: September 15, 2017

Accepted: October 10, 2017

Published: October 13, 2017

Copyright $\odot 2017$ by authors and Scientific Research Publishing Inc. This work is licensed under the Creative Commons Attribution International License (CC BY 4.0).

http://creativecommons.org/licenses/by/4.0/

(c) (i) Open Access

\begin{abstract}
Hyperbaric Oxygen Therapy (HBOT) is a medical procedure and therapeutic modality that utilizes $100 \%$ oxygen. HBOT has been used various medical conditions such as progressive necrotizing fasciitis, peripheral arterial insufficiency, and diabetic wounds of the lower extremities. The case report reported here describes the usage of HBOT as an adjunctive "rescue" measure to salvage a patient's cranioplasty scalp flap after flap ischemia was noted 2 days post-operatively. Patient is a 54 year-old caucasian female who presented to our facility with symptoms of a left MCA infarct. CT and MRI of her brain revealed a left MCA infarct with hemorrhagic stroke with significant cerebral edema, and midline shift. She was taken to the operating room for a left decompressive hemi-craniectomy. She had cranioplasty utilizing native bone flap delayed fashion. She had wound infection after cranioplasty which required removal of native bone. After appropriate treatment for infection, she had cranioplasty utilizing prosthetic Biomet implant. Post-cranioplasty, patient developed ischemic cranial flap. This was recognized within $48 \mathrm{hrs}$ and HBOT was implemented. She has a successful rescue of the ischemic cranial flap after she received 14 consecutive treatments over two weeks. In our knowledge, this is the first successful treatment of HBOT reported after prosthetic cranioplasty for scalp flap ischemia.
\end{abstract}

\section{Keywords}

Hyperbaric Oxygen Therapy, Ischemic Cranial Skin Flap

\section{Background and Importance}

Hyperbaric Oxygen Therapy (HBOT) is a medical procedure and therapeutic 
modality that utilizes $100 \%$ oxygen, administered in a specialized chamber that is set at a pressure greater than atmospheric pressure [1] to assist in the treatment of various medical disorders. The increased oxygen pressure raises the tissue-cellular oxygen diffusion gradient, causing tissue, and ultimately cellular oxygenation to improve [2]. The hyperoxia that occurs due to the use of a hyperbaric oxygen treatment makes this procedure effective when treating various types of medical conditions. Because HBOT has been shown to have an antimicrobial effect on certain bacteria and increases the oxygenation of hypoxic wound tissues one current application is in the treatment of diabetic foot ulcers [3]. The case report reported here describes the usage of hyperbaric oxygen therapy as an adjunctive "rescue" measure to salvage a patient's cranioplasty scalp flap after flap ischemia was noted 2 days post-operatively. In our knowledge, this is the first successful treatment of HBOT reported after prosthetic cranioplasty and scalp flap ischemia.

\section{Clinical Presentation}

$\mathrm{KB}$ is a 54 year-old Caucasian female who presented to our facility with symptoms of a left MCA infarct. CT and MRI of her brain revealed a left middle cerebral artery (MCA) infarct with hemorrhagic conversion, significant cerebral edema, and midline shift. She was taken to the operating room for a left decompressive hemi-craniectomy. She subsequently had significant neurological examination improvement and was discharged to rehab. Nine weeks later, she presented to our emergency department with purulent drainage from her cranial incision. Imaging revealed an epidural abscess, and she was taken for debridement of her craniectomy site. Pan sensitive S. aureus was cultured from the wound, and she was treated with Nafcillin for 6 weeks. 10 weeks after the second operation she was taken back to the operating room for a left cranioplasty with a Biomet implant. She was subsequently discharge but returned 9 weeks later with dehiscence of her incision with exposed hardware. She was thus taken back to the operating room for a second debridement and removal of her prosthesis. Six months later, she returned for her second cranioplasty with a Biomet implant, with a successful closure. Given her substantial cranial flap retraction (Figure 1), burn and wound specialist acute care surgical team was involved during this second cranioplasty procedure. A $7 \mathrm{~mm}$ flat drain with a Jackson Pratt bulb was left in the subgaleal space to drain potential collections. Capillary refill around the incision after closure was seen to be less than 2 seconds. The surgical drain was left in place for 48 hours. The drain was removed but there was noted to be an area of duskiness of the incision where the drain had been tunneled (Figure 2(a) and Figure 2(b)). At this point, concern for flap failure and re-infection of her hardware was high, and so hyperbaric oxygen treatment was recommended which was begun the following day. She received 14 consecutive treatments over the next two weeks and 6 more treatments over the 2 weeks after that (a total of 20 treatments). Each HBOT consisted of 1 oxygen period with an ATA pressure 


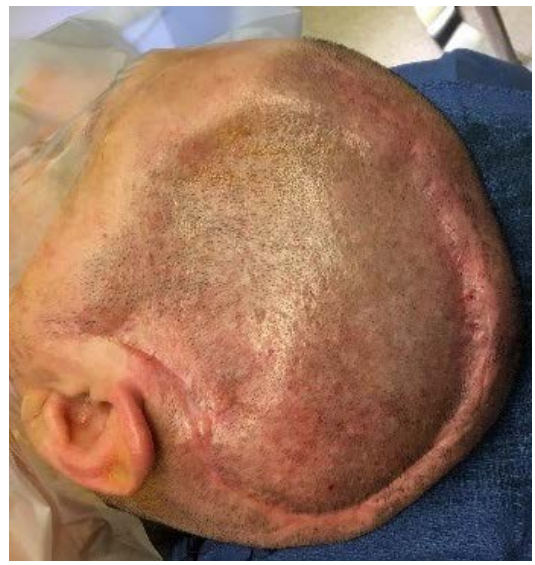

Figure 1. Pre-cranioplasty photograph showing cranial flap retraction.

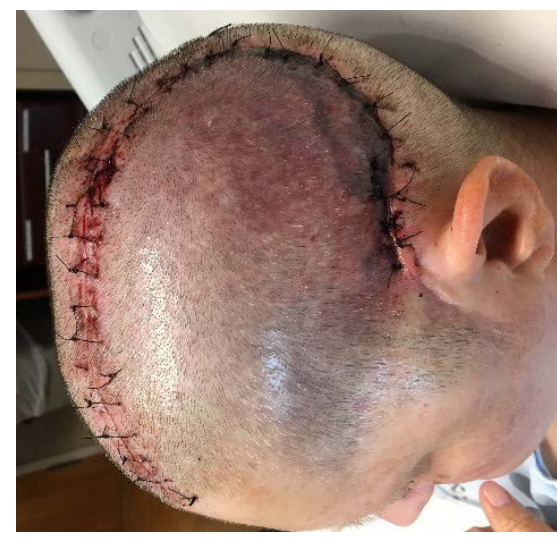

(a)

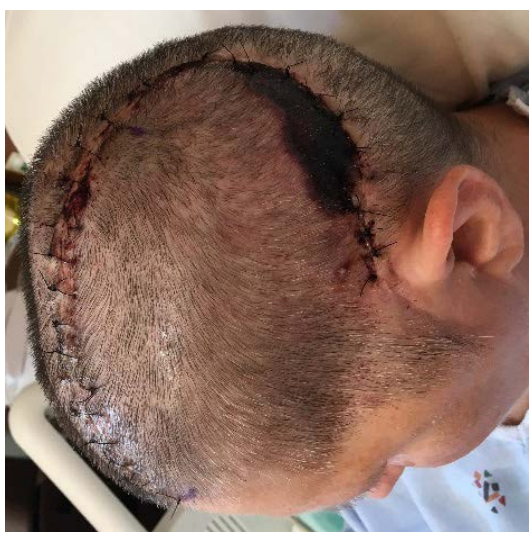

(b)

Figure 2. Post-cranioplaty photograph showing (a) early sign of ischemicand; (b) devitalized cranial flap in the posterior aspect of the cranial incision.

of 2.0 and an oxygen period duration of 90 minutes. Subsequently, she was discharged to home with continued local wound care. Over the following 6 weeks she was followed closely in the outpatient burn and wound center where her wound showed continual improvement with a completely healed incision and no evidence of infection (Figures 3(a)-(c)).

\section{Discussion}

HBOT was initially designed to combat the effects of acute decompression sickness in deep-sea divers. It has been used as a clinical treatment since the early 1900 's. As an adjunct to wound healing, it has been used since the 1960's [4]. It works by increasing the oxygen saturation to assist with oxygen delivery to compromised tissues, such as in certain wounds. By pressurizing $100 \%$ oxygen to 1.5 to 2.5 atmospheres of pressure, the partial-pressure of dissolved oxygen in tissues can increase to as high as $2000 \mathrm{mmHg}$ (normal being approximately 100 $\mathrm{mmHg}$ at sea level in normal, healthy patients). By increasing oxygen delivery to these threatened or compromised tissues, it is postulated that macrophage oxygen-dependent killing efficacy is increased, enhancing infection clearance. It is 


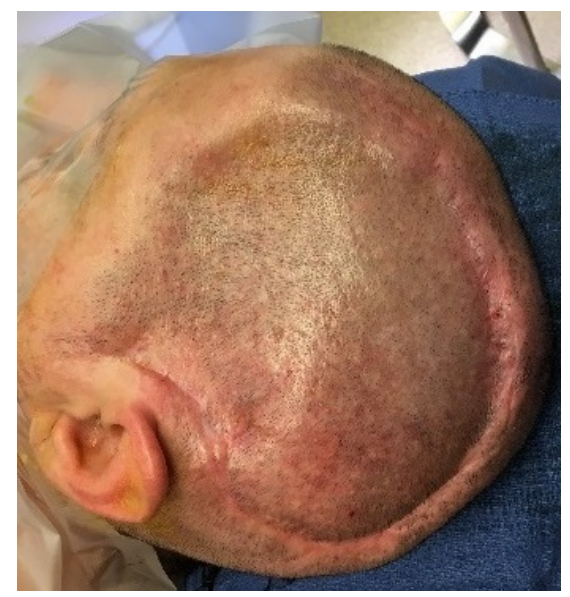

(a)

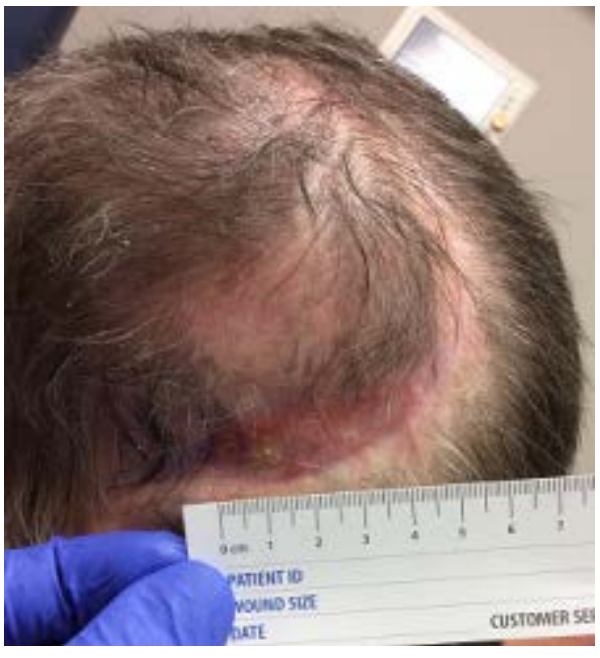

(b)

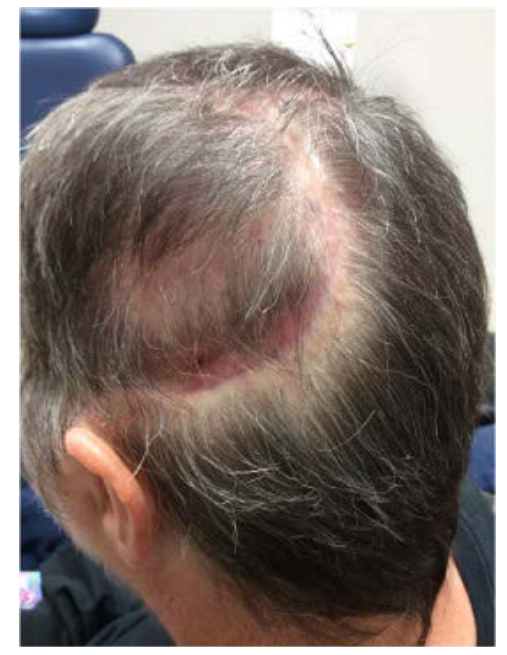

(c)

Figure 3. Photograph of recovering threatened ischemic cranial flap (a) six week; (b) Three months; and (c) six months after completing HBOT.

also theorized that HBOT stimulates both collagen synthesis as well as angiogenesis and enhances fibroblast function, leading to quicker and more robust wound healing. Supranormal oxygenation of tissues also tends to lead to microcirculatory vasodilatory effects, further increasing their oxygenation and the resultant salutary and pleiotropic effects of increased oxygenation of tissues, cell lineages, and oxygen-dependent antibiosis. In addition, the supranormal but localized delivery of oxygen to compromised tissues has been shown in both animal and clinical studies to contribute to both flap as well as skin graft success [5]. According to the Center for Medicaid and Medicare Services (CMMS), indications for its usage in wounds include compromised grafts or flaps [6]. Therefore, if a hyperbaric chamber was available, any patient with a threatened graft or flap without contraindications (such as uncontrolled seizures, severe uncontrolled asthma, presence of pneumothorax, or relative contraindications such as severe claustrophobia (assuming a monoplace chamber) or Eustachian tube dysfunction) could benefit from its usage. 


\section{Conclusion}

In our patient, early recognition of her "threatened ischemic cranial flap" and implementation of inpatient hyperbaric therapy successfully saved cranial skin flap. In addition, the procedure prevented the underlying prosthesis from contamination and subsequent need for removal and later free-flap reconstructive techniques of salvage.

\section{References}

[1] Gill, A.L. and Bell, C.N. (2004) Hyperbaric Oxygen: Its Uses, Mechanisms of Action and Outcomes. QJM, 97, 385. https://doi.org/10.1093/qjmed/hch074

[2] Leach, R.M., Rees, P.J. and Wilmshurst, P. (1998) Hyperbaric Oxygen Therapy. British Medical Journal, 317, 1140-1143. https://doi.org/10.1136/bmj.317.7166.1140

[3] Londahl, M., Katzman, P., Nilsson, A. and Hammarlund, C. (2010) Hyperbaric Oxygen Therapy Facilitates Healing of Chronic Foot Ulcers in Patients with Diabetes. Diabetes Care, 33, 998-1003. https://doi.org/10.2337/dc09-1754

[4] Boateng, J. and Catanzano, O. (2015) Advanced Therapeutic Dresings for Effective Wound Healing-A Review. Journal of Pharmaceutical Sciences, 104, 3653-3680. https://doi.org/10.1002/jps.24610

[5] Baynosa, R. and Zambone, W.A. (2012) The Effect of Hyperbaric Oxygen on Compromised Flaps. Undersea \& Hyperbaric Medicine, 39, 857-865.

[6] Centers for Medicare \& Medicaid Services (2006) Manual Section Number 20.29: Hyperbaric Oxygen Therapy. In: Medicare National Coverage Determinations Manual.Version Number 3, US Department of Health and Human Services, Washington DC. 